\title{
Article \\ Suppression of Acoustic Resonances in BST-Based Bulk-Ceramic Varactors by Addition of Magnesium Borate
}

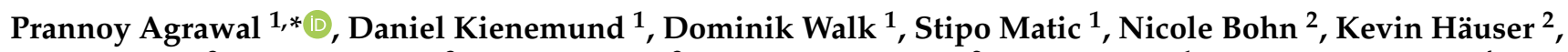 \\ Thomas Fink $^{3}$, Mike Abrecht ${ }^{3}$, Walter Bigler ${ }^{3}$, Joachim R. Binder ${ }^{2}$, Rolf Jakoby ${ }^{1}$ and Holger Maune ${ }^{4}$ \\ 1 Institute of Microwave and Photonics, Technical University of Darmstadt, 64283 Darmstadt, Germany; \\ kienemund@imp.tu-darmstadt.de (D.K.); dominik.walk@tu-darmstadt.de (D.W.); \\ stipo.matic@tu-darmstadt.de (S.M.); rolf.jakoby@tu-darmstadt.de (R.J.) \\ 2 Institute for Applied Materials, Karlsruhe Institute of Technology, 76021 Karlsruhe, Germany; \\ nicole.bohn@kit.edu (N.B.); kevin.haeuser@kit.edu (K.H.); joachim.binder@kit.edu (J.R.B.) \\ 3 COMET AG, 3175 Flamatt, Switzerland; thomas.fink@comet.ch (T.F.); mike.abrecht@comet.ch (M.A.); \\ walter.bigler@comet.ch (W.B.) \\ 4 Microwave and Communication Engineering, Otto von Guericke University Magdeburg, \\ 39106 Magdeburg, Germany; holger.maune@ovgu.de \\ * Correspondence: prannoy.agrawal@tu-darmstadt.de
}

check for updates

Citation: Agrawal, P.; Kienemund, D.; Walk, D.; Matic, S.; Bohn, N.; Häuser, K.; Fink, T.; Abrecht, M.; Bigler, W.; Binder, J.R.; Jakoby, R.; Maune, H. Suppression of Acoustic Resonances in BST-Based

Bulk-Ceramic Varactors by Addition of Magnesium Borate. Crystals 2021, 11, 786. https://doi.org/ $10.3390 /$ cryst11070786

Academic Editors: Caroline Borderon, Raphaël Renoud, Hartmut W. Gundel and Kevin Nadaud

Received: 11 June 2021

Accepted: 28 June 2021

Published: 6 July 2021

Publisher's Note: MDPI stays neutral with regard to jurisdictional claims in published maps and institutional affiliations.

Copyright: (c) 2021 by the authors. Licensee MDPI, Basel, Switzerland. This article is an open access article distributed under the terms and conditions of the Creative Commons Attribution (CC BY) license (https:// creativecommons.org/licenses/by/ $4.0 /)$.

\begin{abstract}
This work presents a method for reducing acoustic resonances in ferroelectric barium strontium titanate (BST)-based bulk ceramic varactors, which are capable of operation in highpower matching circuits. Two versions of parallel-plate varactors are manufactured here: one with pure BST and one with 10 vol-\% magnesium borate, $\mathrm{Mg}_{3} \mathrm{~B}_{2} \mathrm{O}_{6}(\mathrm{MBO})$. Each varactor includes a 0.85-mm-thick ferroelectric layer. Acoustic resonances that are present in the pure BST varactor are strongly suppressed in the BST-MBO varactor and, hence, the Q-factor is increased over a wide frequency range by the addition of small amounts of a low-dielectric-constant (LDK) MBO. Although the tunability is reduced due to the presence of non-tunable $\mathrm{MBO}$, the increased Q-factor extends the varactor's availability for low-loss and high-power applications.
\end{abstract}

Keywords: barium strontium titanate (BST); magnesium borate; acoustic resonances; electrostriction; bulk-ceramics

\section{Introduction}

As minimum structure sizes reach atomic scale boundaries, the focus is on improving the manufacturing processes of integrated circuits. These are mostly based on plasmaenhanced deposition or etching [1]. The lower industrial, scientific and medical (ISM) band frequencies, especially at $13.56 \mathrm{MHz}$, are used for this purpose. The plasma processes need a tunable matching circuit to match the impedance of the plasma chamber to the impedance of the radio frequency $(\mathrm{RF})$ power generator during the plasma ignition and operation intervals. Traditionally, mechanically tuned vacuum capacitors are used, which have limited response times of around $1 \mathrm{~s}$ for a relative maximum change in capacitance. As the thickness of the processed layers decreases, the plasma process duration also decreases, pushing the required response time to the sub-second regime [2].

In general, semiconductor varactor diodes, PIN-diode switched capacitor banks, microelectromechanical systems (MEMS)-based solutions, and varactors based on functional materials such as ferroelectrics have been implemented. The semiconductor varactor diodes suffer from a low Q-factor, low linearity and poor power handling [3,4], and the PIN-diode switched capacitor banks lack continuous tuning, as they provide discrete capacitances only [3]. MEMS-based solutions have been shown to be promising alternative but there is still scope of improvement in terms of power handling and mechanical reliability under hot switching conditions [3,5], which are essential for plasma applications. 
Ferroelectric varactors based on barium strontium titanate (BST) provide a fast, high and continuous tunable permittivity with high linearity. BST-based varactors also possess a high breakdown field strength in the $\mathrm{kV} / \mathrm{mm}$ region. These varactors can be based on three types of technology - thin-film, thick-film and bulk-ceramic. The latter two technologies are more suitable for high-power applications [6,7]. The stoichiometric ratio of barium to strontium determines the operating temperature, which makes it important for the BST to be in the paraelectric phase to maintain centrosymmetry [8]. However, the main drawback of BST, as a solid-state dielectric, is its relatively high dielectric loss, which limits the varactor's extensive use [9,10]. Another disadvantage is the electric-field-induced electrostrictive strain across the ferroelectric layer, which results in acoustic waves that are reflected from adjacent layers, reducing the varactor's Q-factor. In thin-film devices, acoustic resonances are a well-known phenomenon [11,12] and, recently, their presence in thick-film applications has also been observed in the lower $\mathrm{MHz}$ range [7]. Going forward, acoustic resonances methodology and a method for their suppression in BST-based bulkceramic varactor will be discussed using the composite ceramic approach.

\section{Acoustic Resonances Methodology}

\subsection{Theory}

Assuming a parallel-plate varactor with a bulk ferroelectric layer between two thick electrodes, strain is induced across the layer with the application of an electric-field. According to $[13,14]$, this strain is represented as

$$
S=Q P^{2}(E)
$$

where $Q$ is the electrostrictive coefficient and $P(E)$ is the polarization due to the electricfield $E$ across the layer. For the characterization of functional materiasl, such as BST, small-signal analysis is performed with a large bias signal $E_{T u n}$ to tune the material layer. This large bias signal is mainly responsible for the $\mathrm{P}(\mathrm{E})$ and, therefore, the strain can be simply expressed as

$$
S=Q P^{2}\left(E_{\text {Tun }}\right) \propto Q \varepsilon^{2} E_{\text {Tun }}^{2}
$$

where $\varepsilon$ is the BST layer's permittivity. As $Q$ is inversely proportional to the permittivity $\varepsilon$ [14], Newnham(1997), (2) modifies to

$$
S \propto \varepsilon E_{\text {Tun }}^{2}
$$

which suggests that the strain is directly dependent don the permittivity of the material, aside from the bias fields.Finally, the induced piezoelectricity $g$ can be expressed as

$$
g=\frac{\partial S}{\partial P(E)}=2 Q P\left(E_{T u n}\right) \propto 2 Q \varepsilon E_{T u n}
$$

which is responsible for the presence of acoustic in the small AC signal.

Physically, the displacement of the $\mathrm{Ti}^{4+}$ ions in the BST crystal with the application of bias fields leads to the above-mentioned strain $S$. The ionic displacement generates proportional mechanical energy. This mechanical energy generates acoustic waves and part of this energy is reflected back to the BST layer at interfaces to adjacent layers, forming standing waves or acoustic resonances. Simultaneously, due to energy conservation, the acoustic waves lead to a decrease in the varactor's Q-factor or increase in equivalent series resistance (ESR) [15]. Generally, the acoustic resonances are harmonic in nature, which are spread over a frequency range, leading to the equivalent harmonic drops of the Q-factor or spikes in ESR in this frequency range. According to Equation (3), the proposed solution is to introduce a small amount of low-dielectric-constant (LDK) material to the BST layer to reduce the relative permittivity of the BST layer, with a slight decrease in tunability. This eventually reduces electrostrictive strain and, therefore, the effects of induced piezoelectricity. Hence, the effects of standing acoustic waves can be minimized. 


\subsection{Simulative Analysis}

Considering the computational complexity associated with the 3D simulation environment, a 2D piezoelectric/acoustic simulation environment is set up in COMSOL Multiphysics, as shown in Figure 1a, where a BST bulk of $2 \times 1 \mathrm{~mm}^{2}$ cross-section is placed between two silver electrodes to form a parallel-plate varactor. In the third dimension, the varactor is assumed to be uniform, forming plane-symmetric geometry. The model is in the strain-charge form of the piezoelectric effect, computing the structural strain $S$ from the applied electrical field $E$ to investigate the electrostrictive effects of the BST-based varactors, as mentioned in [16]. The chosen elastic compliance is marginally higher in comparison, at around $6.35 \times 10^{-12} \mathrm{~Pa}^{-1}$ [17], to compensate for the lower strontium content in the BST used in this work, which is discussed later in Section 3. In the other varactor configuration, a small amount of BST is replaced by the LDK inclusions, as shown in Figure $1 b-d$, by the blue-colored circles. Each inclusion has $0.16 \mathrm{~mm}$ diameter, which is based on the observations from the Figure 2d. Due to the lack of knowledge on MBO's mechanical parameters, the material for LDK inclusions is chosen to be COMSOL predefined linear elastic material, Alumina $\left(\mathrm{Al}_{2} \mathrm{O}_{3}\right)$, with a dielectric contrast of around 1:350 with BST. The permittivity of BST is taken to be around 2000 [18]. Three configurations of 5, 10 and 30\% LDK inclusion compositions are chosen and 100 simulations for each configuration with different non-overlapped random arrangements of LDK inclusions are carried out to prove its reliability in the acoustic suppression of the model with inclusions. The random arrangements are based on the uniform distribution of probabilities of the position of LDK inclusions across horizontal and vertical direction within the ferroelectric layer. These simulations are based on the Monte-Carlo method to estimate the effects of different locations of inclusions in the ferroelectric layer. It is to be noted that the dielectric loss of all materials is ignored here, to focus on the effects of the low dielectric constant and related mechanical properties of LDK inclusions.

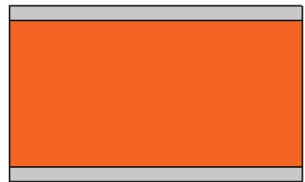

(a)

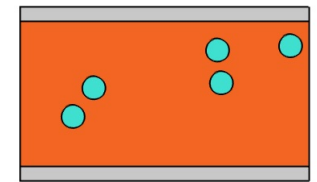

(b)

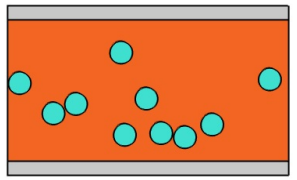

(c)

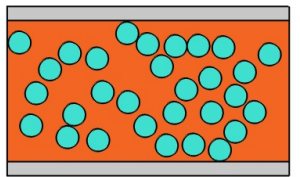

(d)

Figure 1. 2D simulation model design with BST (orange) between two silver electrodes (gray) with randomly placed LDK inclusions (blue): (a) without the inclusions and (b-d) with 5\%, 10\%, 30\% LDK inclusions in the BST region. The BST is $2 \times 1 \mathrm{~mm}^{2}$ in dimensions and each inclusion is $0.16 \mathrm{~mm}$ in diameter.

The extracted ESR for the model without inclusions is compared with the mean $\mu$ of all 100 simulations of each configurations of model with inclusions in Figure 3. The model without inclusion, shown with the squared black line, depicts the presence of acoustics at around $11.3 \mathrm{MHz}, 15.7 \mathrm{MHz}, 19.9 \mathrm{MHz}$ and $24.1 \mathrm{MHz}$ with ESR peaks. The significant reduction in ESR, for the peak around $15.7 \mathrm{MHz}$ and $19.9 \mathrm{MHz}$, from around $200 \Omega$ to under $25 \Omega$ for all configurations of inclusions, shows a considerable suppression of acoustics. On the contrary, the $\mu$ comparison does not show a reliable suppression for all the simulations of all configurations, as there can be a few outlier simulations, which do not show noticeable suppression. Hence, the variance study becomes critical in ensuring the model's reliability towards acoustic suppression. 


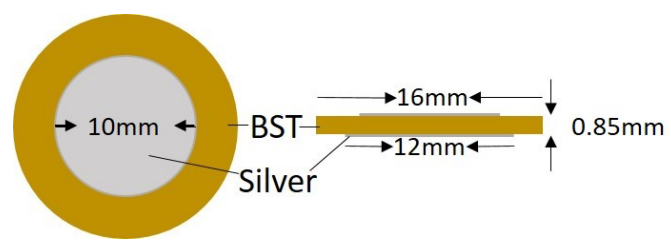

(a)

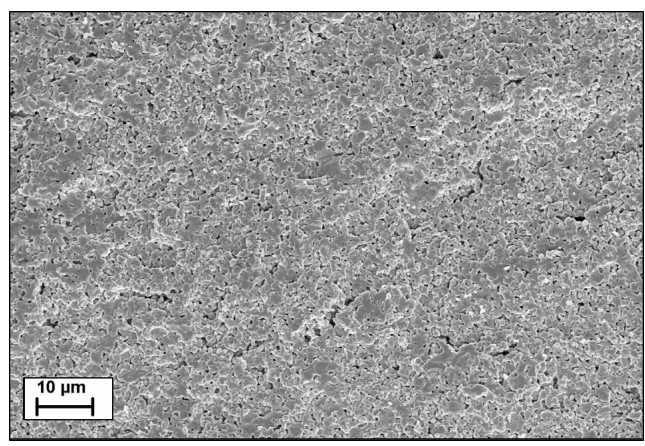

(c)

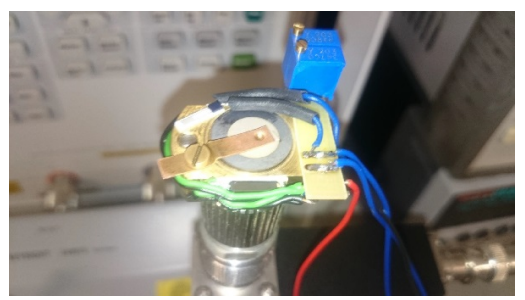

(e)

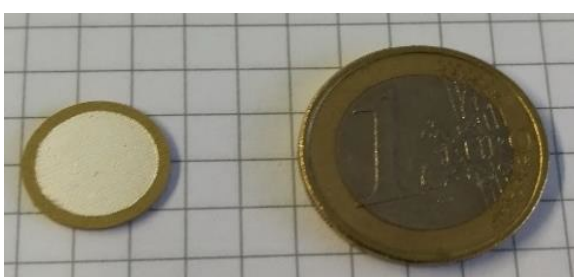

(b)

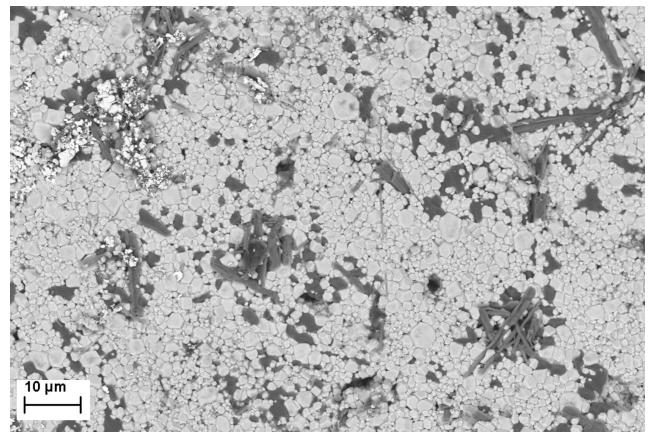

(d)

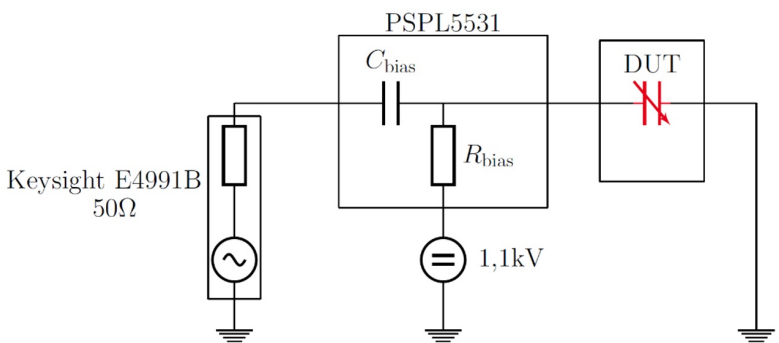

(f)

Figure 2. Manufactured varactor pellet with (a) schematic (top and side view) and (b) realized varactor. Microstructure analysis with scanning electron microscope images of (c) pure BST varactor (d) BST-MBO varactor. In the BST-MBO varactor, BST is shown by white color and MBO phases are shown by gray color. The measurement approach is shown with (e) pellet holder and (f) schematic circuit of the setup.

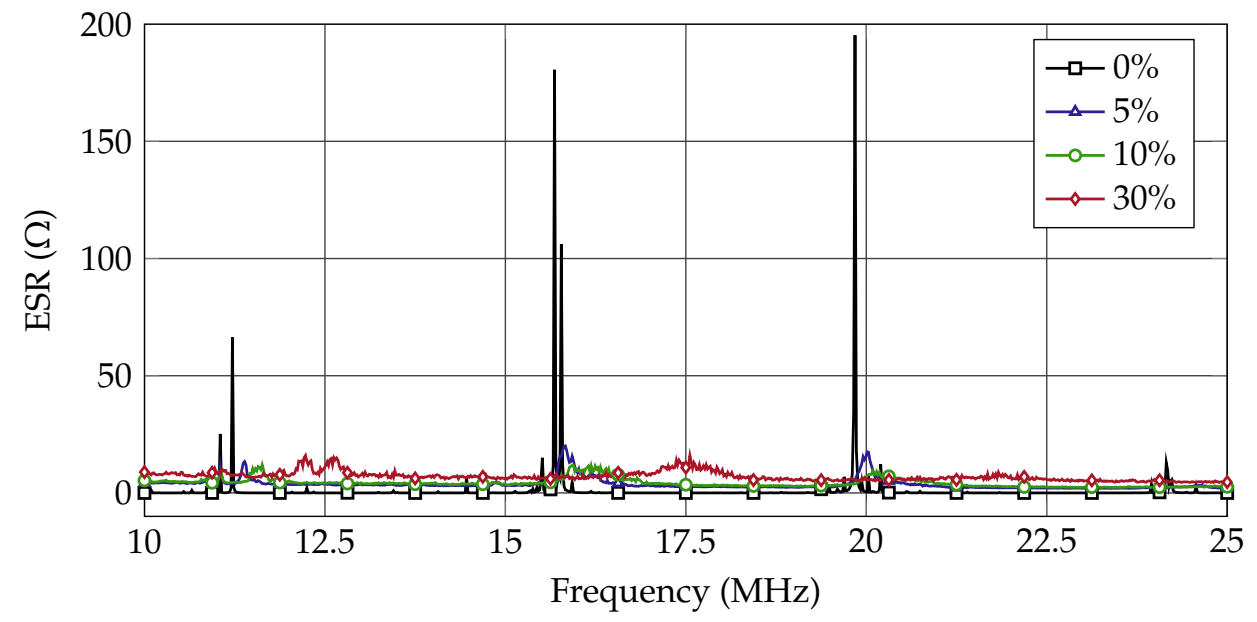

Figure 3. Comparison of mean $\mu$ ESR extracted from 2D simulation model with increasing composition percentages of inclusions. Considerable suppression of acoustics is observed here, with a tolerable increase in ESR at non-acoustic frequencies.

The cumulative variance $\sigma^{2}$ of the extracted ESR for 10\% LDK inclusions is shown in Figure 4a. It can be observed that, with the increasing number of simulations with different 
arrangements of LDK inclusion, the spread of extracted ESR reduces and the simulation environment becomes saturated with further changes in the spread of ESR. It should be noted that the narrow bandwidth of acoustics and small shifts in acoustic frequencies with different arrangements are the reason for the high variance here. Comparing the $\sigma^{2}$ over 100 simulations, searching for different inclusion compositions, as shown in Figure $4 b, \sigma^{2}$ is the minimum for a $10 \%$ inclusion configuration. This denotes that the $10 \%$ inclusion configuration shows less influence from microstructural changes with the different arrangement of inclusions. Finally, the extracted ESR for the model without inclusions is compared with the statistically adjusted extracted ESR of each model configuration, with inclusions shown in Figure 5. For comparison, the upper bound limit of the extracted ESR from the model with inclusions is considered. The upper bound is stated by the $\mu$ of 100 simulations with the addition of $3 \sigma$, assuming the extracted ESR varies according to normal distribution. This upper bound limit, statistically, considers the $99 \%$ confidence interval of the extracted ESR values for each frequency point in all the simulations considered.

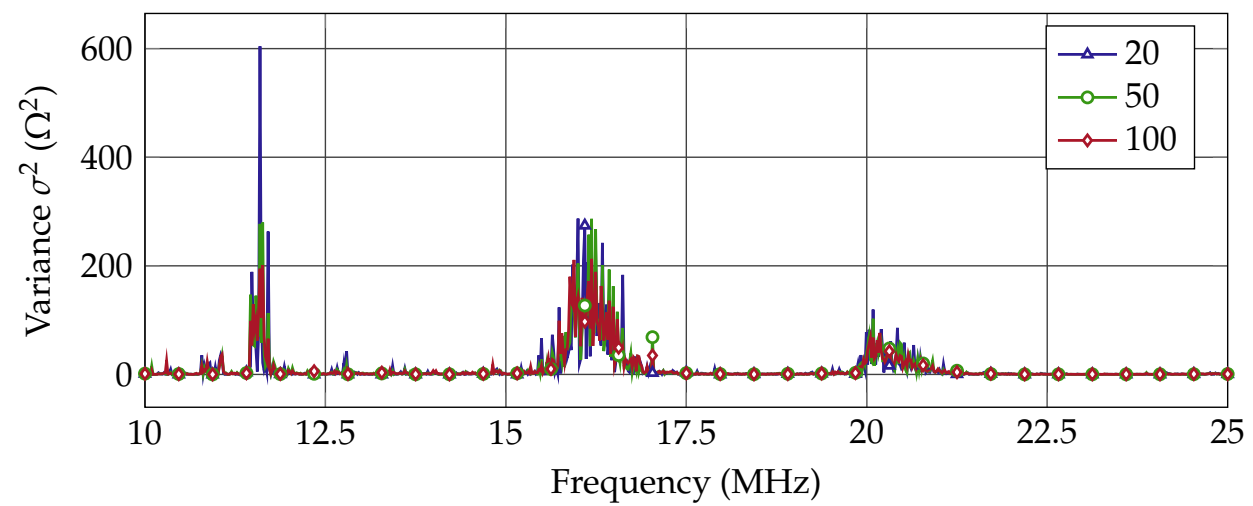

(a) 10\% inclusions with varying no. of simulations.

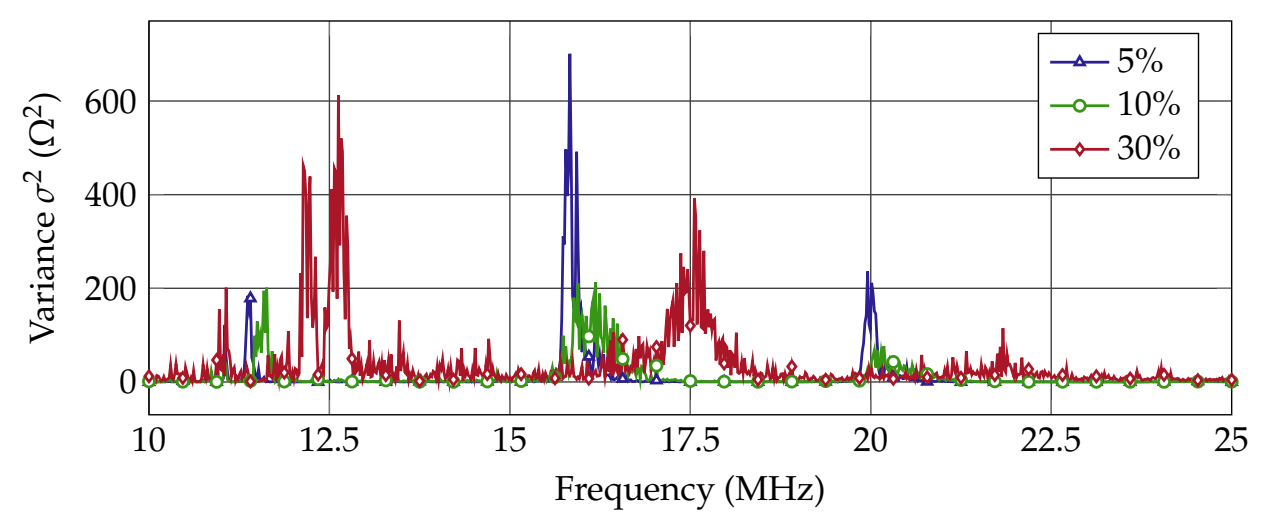

(b) Varying inclusions content with 100 simulations each.

Figure 4. Comparison of extracted simulated ESR statistics from 2D simulation models of different inclusions with the first 20,50 and 100 simulations. (a) shows the variance in the spread of the $10 \%$ inclusion configuration, depicting the model saturation of variation in extracted ESR with the increasing number of simulations. (b) compares the variance for 5, 10 and 30\% LDK inclusions for 100 simulations in each configuration, which shows that, with increasing LDK inclusions, the acoustics suppression increases, but decreases again with a further increase in inclusions. 


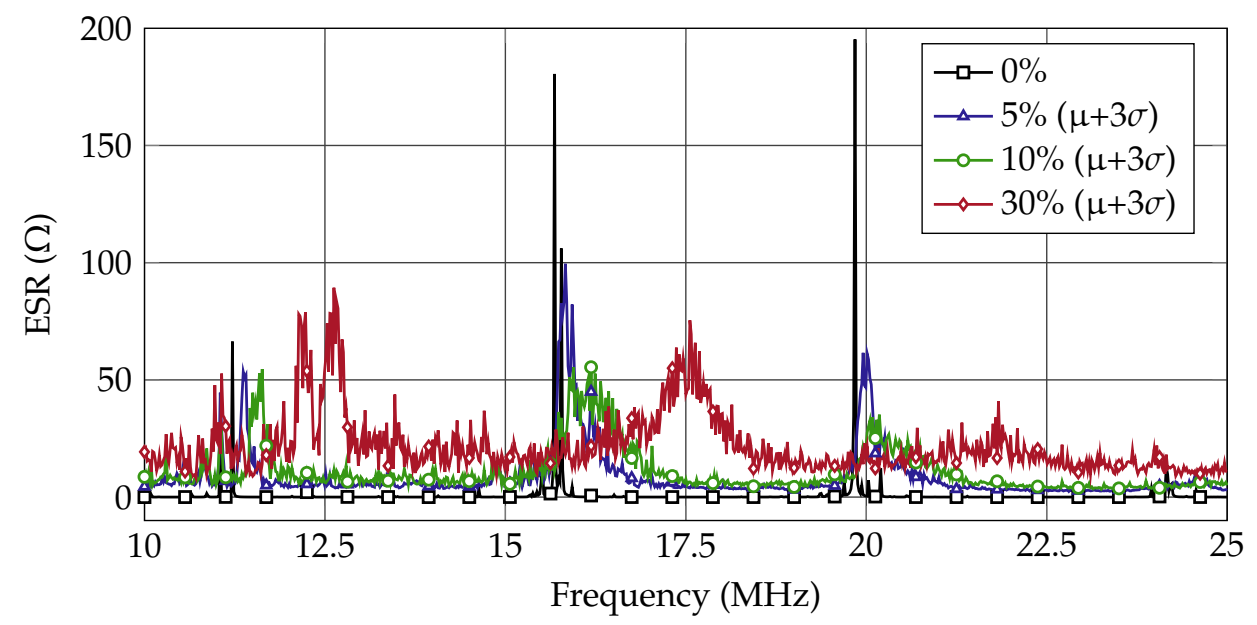

Figure 5. Comparison of ESR extracted from the 2D simulation model with increasing composition percentages of inclusions. Here, the upper bound limit ( $99 \%$ confidence interval) of ESR from mean and standard deviation is considered, and the lower bound is ignored. Considerable suppression of acoustics is observed here, with a tolerable increase in ESR at non-acoustic frequencies. A shift in frequencies of acoustics to higher frequencies is also observed with increasing amounts of LDK inclusions.

The reduction in ESR, for the peak at around $15.7 \mathrm{MHz}$ and $19.9 \mathrm{MHz}$, from around $200 \Omega$ to $100 \Omega$ for all configurations of inclusions, ensures the considerable suppression of acoustics. This suppression effect of around $11.3 \mathrm{MHz}$ and $24.1 \mathrm{MHz}$ is limited due to microstructural changes, with different arrangements for all inclusion configurations; however, with the $30 \%$ LDK inclusions, this limitation is more prominent. In conclusion, when comparing all the inclusion configurations, the extracted ESR and microstructural influence with different arrangements is lower for the $10 \%$ inclusion configuration than the other inclusion configurations for the whole range of considered frequencies. In fact, a trend of the increasing and then decreasing suppression of acoustics with the increase in LDK inclusions is witnessed here. This leads to the $10 \%$ inclusions configuration being selected as the most appropriate choice for the experiment, among the three configurations of inclusions considered.

As the realized varactor is in different geometric form, as shown in Figure $2 b$, the model geometry is modified, similar to the realized varactor, which is shown in Figure 6. The modified geometry becomes more complex and requires more inclusions to cover the required inclusion composition in the BST region. The realized varactor's widest cut slice, as shown in Figure 2a, is assumed for the 2D simulation environment, to consider the effects of its crucial dimensions. The suppression behaviour is already shown in the 2D model above and, hence, only $10 \%$ LDK inclusion is considered in the modified model. This modified model is simulated with $10 \%$ inclusions of LDK material to demonstrate the acoustic suppression behaviour expected in the realized varactor geometry. The comparison of the simulated ESR of both, with inclusions and without inclusions, is shown in Figure 7. Here, the upper bound limit of the extracted ESR for the modified model with inclusions is compared with the modified model without inclusions. The main advantage with the modified model is the position of the acoustics in the frequency range, which is expected to correctly match the extracted measured ESR. The distinct acoustic resonances are observed at around 9.9 MHz, 16.5 MHz and 23.1 MHz. 


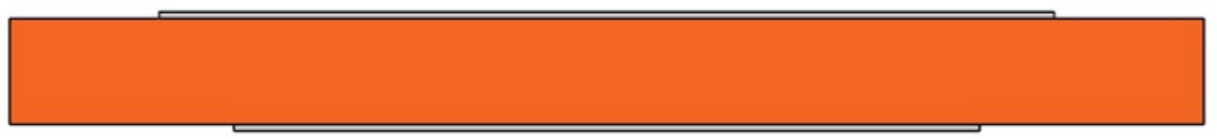

(a)

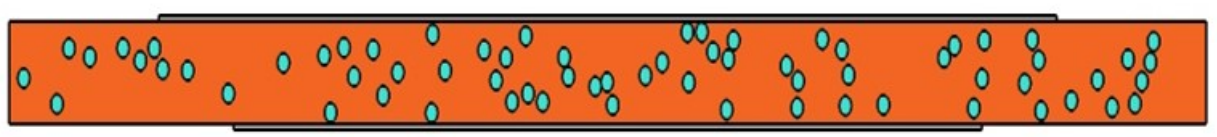

(b)

Figure 6. Modified 2D simulation model to adapt the realized varactor. $(\mathbf{a}, \mathbf{b})$ are the model without and with $10 \%$ LDK inclusions (blue) respectively. The electrodes are $0.01 \mathrm{~mm}$ thick and the BST (orange) region is $16 \times 0.85 \mathrm{~mm}^{2}$ in dimensions close to the measured dimensions of the realized varactors. Each inclusion is $0.16 \mathrm{~mm}$ in diameter.

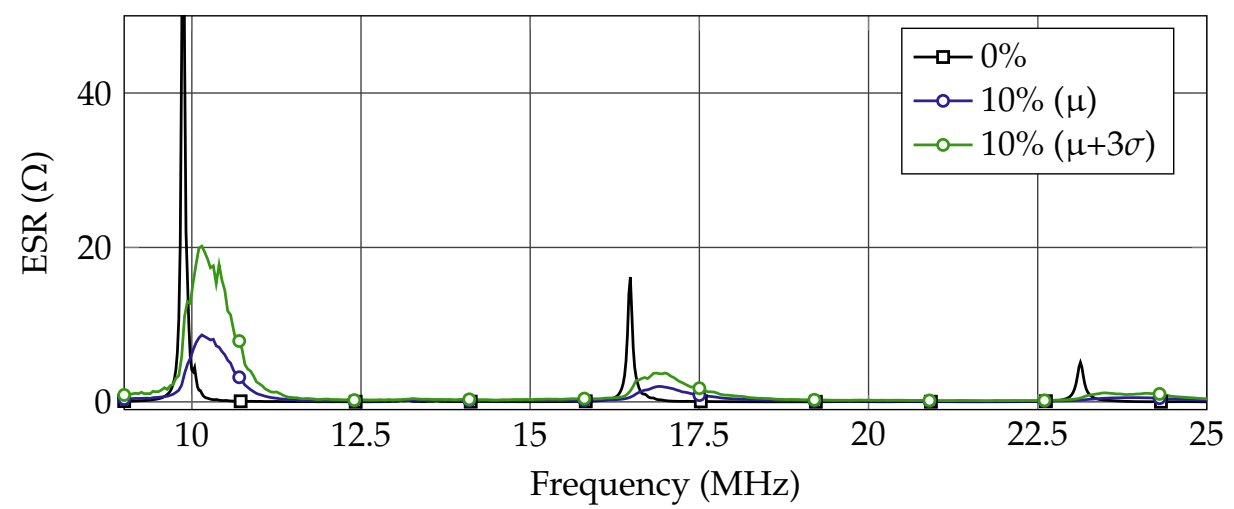

Figure 7. Comparison of extracted simulated ESR statistics from a modified 2D simulation model of $10 \%$ inclusions. The upper bound limit $\mu+3 \sigma$ and mean $\mu$ of extracted ESR of $10 \%$ LDK inclusion configuration for 80 simulations is compared with no-inclusion model and a similar acoustics suppression is witnessed as compared to the $2 \mathrm{D}$ simulation model discussed earlier.

With the simulation environment showing a considerable suppression of acoustics with the addition of small amounts of LDK material, a strong rationale is set up for experimentation and is discussed in the upcoming sections.

\section{Varactor Design and Characterization}

Due to the plasma application requirements for maintaining high operating temperatures of around $40-50{ }^{\circ} \mathrm{C}$, the ratio of barium to strontium in BST is chosen so that its Curie temperature $\left(T_{C}\right)$ is around $20^{\circ} \mathrm{C}$. The difference in temperatures ensures that the BST stays in paraelectric phase, despite the temperature change in the vicinity of the setup. The BST has a relative permittivity of more than 2000 and shows Q-factor of around 100 in the lower $\mathrm{MHz}$ range, as seen in Figure 8a. The low-loss LDK magnesium borate $\mathrm{Mg}_{3} \mathrm{~B}_{2} \mathrm{O}_{6}$ (MBO) is chosen as an additive as it is efficient in reducing dielectric loss [10]. The MBO has a relative permittivity and Q-factor of around 7 and 2500 [18], respectively. Two versions of varactors are manufactured here: one from pure BST and the other from BST with $10 \mathrm{vol}-\% \mathrm{MBO}$. The prepared spray granulates are compressed under high pressure to form solid pellets, using a single-axis manual laboratory press. Each pellet is then sintered and eventually metalized with silver paste to obtain a parallel-plate varactor, as depicted in Figure $2 b$. Figure $2 c$, d show the scanning electron microscope (SEM) images, depicting the microstructural views of the manufactured varactors. 


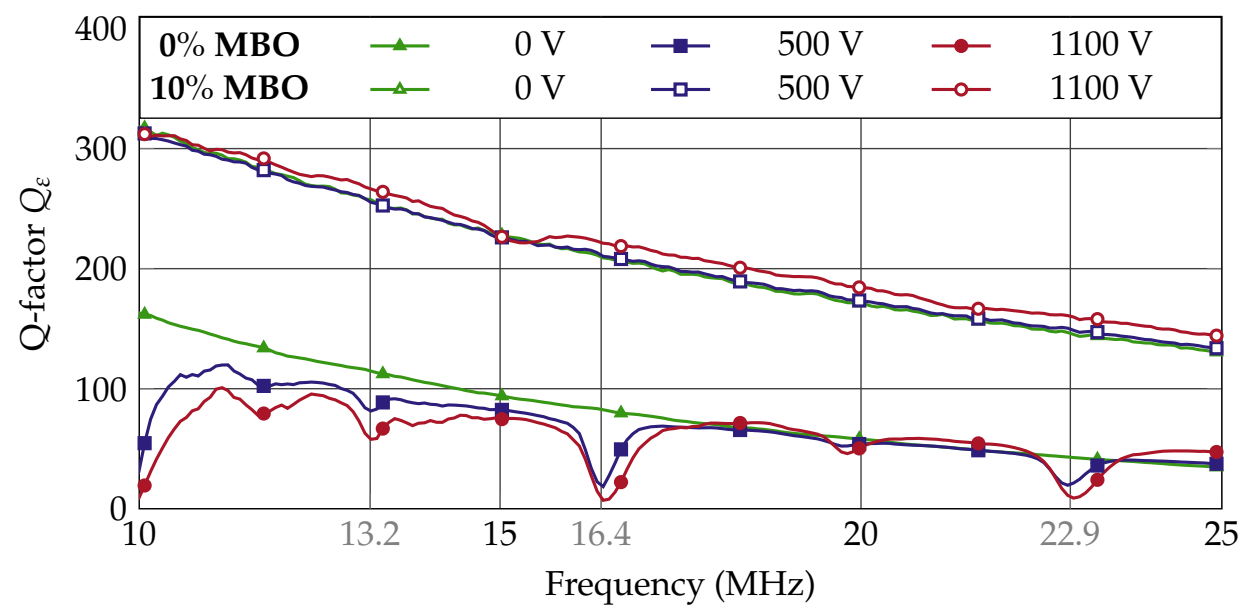

(a)

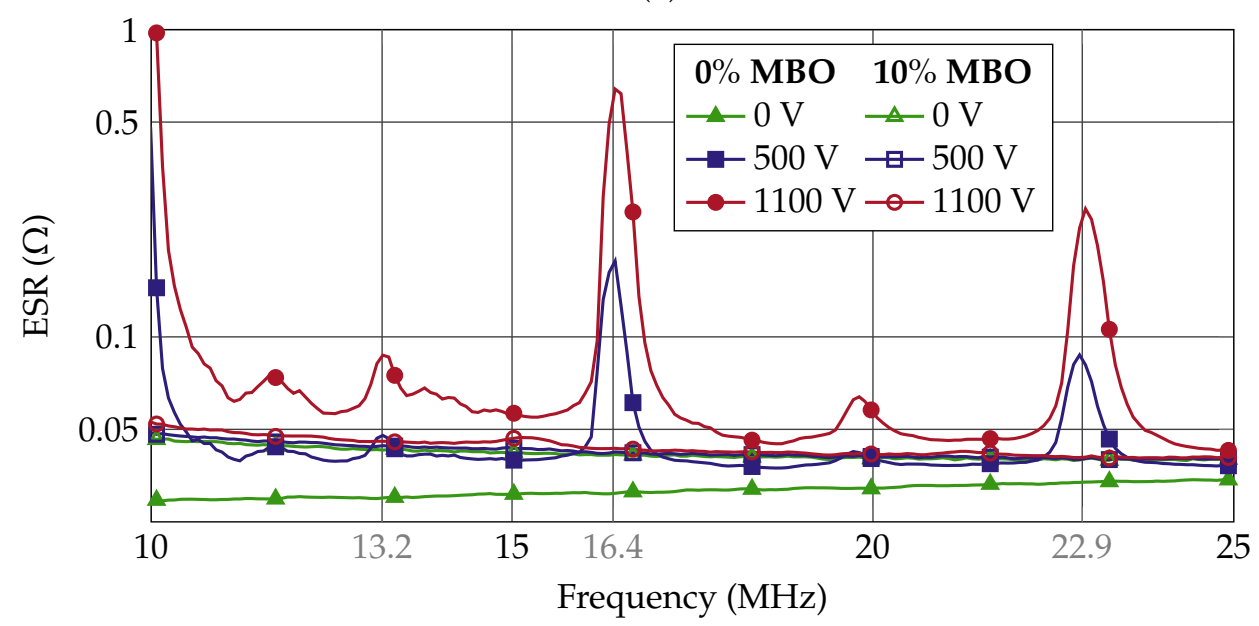

(b)

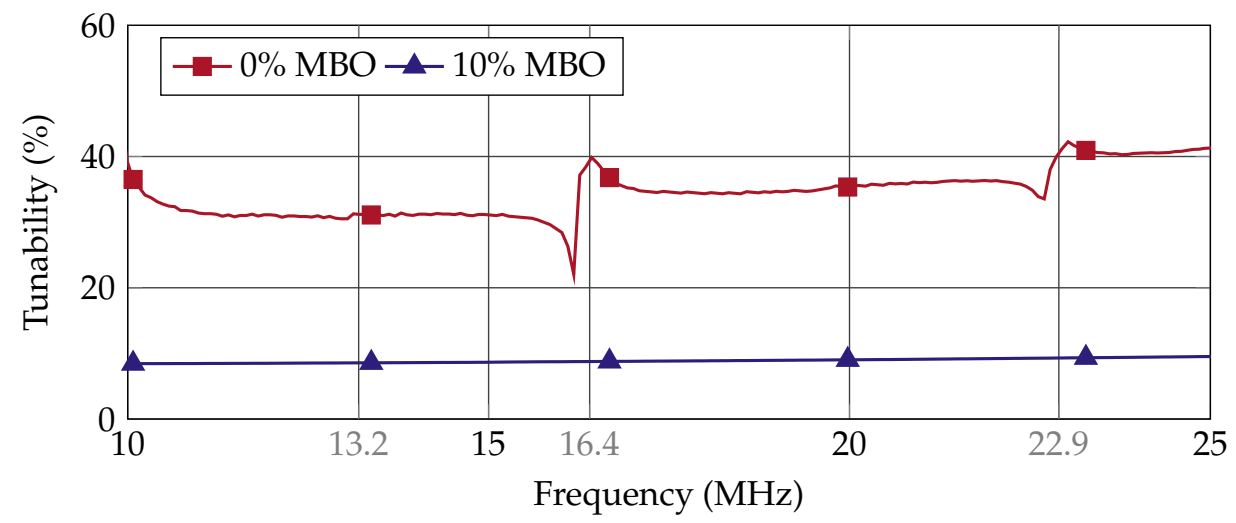

(c)

Figure 8. Extracted material values from measurements: (a) Q-factor, (b) ESR and (c) tunability at $1.1 \mathrm{kV}$.

The electrical characterization of the varactor is performed in a heated pellet holder; see Figure 2e. The holder is based on the APC-7 standard and is heated to maintain $50{ }^{\circ} \mathrm{C}$ on the varactor. Small-signal characterization is performed from $10 \mathrm{MHz}$ to $25 \mathrm{MHz}$ with a Keysight Impedance Analyzer E4991B. The schematic of the measurement circuit is shown in Figure 2f. DC bias voltages up to $1100 \mathrm{~V}$, producing maximum electric fields of around $1.29 \mathrm{kV} / \mathrm{mm}$, are applied using a Keithley 2410 Sourcemeter. Before every measurement, the setup is calibrated by standard short, open and load (SOL) calibration at 
the end of the biasing circuit and the DC bias source was switched on at $0 \mathrm{~V}$. The extracted capacitance, extracted ESR and Q-factor readings of the varactor are taken directly from the Impedance Analyzer.

\section{Evaluation}

Figure 8a,b compare the extracted Q-factor $Q_{\varepsilon}$ and ESR from measured values for both configurations, respectively. A notable presence of acoustics in pure BST varactor can be observed at $13.2 \mathrm{MHz}, 16.4 \mathrm{MHz}$ and $22.9 \mathrm{MHz}$, which decreases the Q-factor with increasing bias voltages. In comparison, an almost complete suppression of acoustics is observed at any bias voltage applied in the BST-MBO varactor. The ESR shows the same behaviour as predicted in the simulation environment earlier, shown in Figure 7. Due to the simplification with no dielectric losses, the simulation model cannot extract the absolute magnitudes of extracted ESR and the comparison can only be made for the relative changes. In terms of the position of acoustics in the frequency range, the distinct acoustics are present at similar frequencies of around $16.4 \mathrm{MHz}$ and $22.9 \mathrm{MHz}$, confirming the simulation model's viability. The difference in the position is around $6.4 \mathrm{MHz}$, which also conforms with the simulation model discussed in Section 2.

Moreover, the unbiased Q-factor is increased from around 120 to 270 at $13.2 \mathrm{MHz}$, denoting the decrease in dielectric loss. The shift in ESR peaks over frequency is not noticeable, which is suspected to be due to the low dielectric loss of $\mathrm{MBO}$, further increasing the Q-factor and reducing the ESR values. Some small dips, such as the one around $15 \mathrm{MHz}$, in the Q-factor, are visible in the BST-MBO varactor, but nothing conclusive can be stated from these. As discussed in [18], the extracted capacitance values are directly proportional to the extracted relative permittivity for the cylindrical disk capacitor used in this work. This leads to the conclusion that the capacitance tunability $\tau_{C}$ equals the permittivity tunability $\tau_{\varepsilon}$ and is defined as

$$
\tau_{\varepsilon}=\frac{\varepsilon(0)-\varepsilon(V)}{\varepsilon(0)}=\frac{C(0)-C(V)}{C(0)}=\tau_{C}
$$

where $\varepsilon(V)$ and $C(V)$ are the relative permittivity and capacitance at voltage $V$, respectively. $\tau_{\varepsilon}$ decreases from around $30 \%$ to $10 \%$, as shown in Figure $8 \mathrm{c}$. The reduction in $\tau_{\varepsilon}$ is observed to be larger than expected, and is certainly not comparable to the elevation in $Q_{\varepsilon}$, which is suspected to be due to the formation of a third non-tunable phase due to ionic exchanges between BST and MBO [19]. The same behavior is also witnessed with the non-biased capacitance, as the value decreases from around $3.58 \mathrm{nF}$ to around $1.11 \mathrm{nF}$ with the introduction of MBO.The figure-of-merit is taken as the material quality factor $(\eta)$ [20], which is defined as

$$
\eta=\min \left(Q_{\varepsilon}\right) \cdot \tau_{\varepsilon}
$$

as shown in the Figure 9a. At $16.4 \mathrm{MHz}$ and $22.9 \mathrm{MHz}$, a significant improvement in $\eta$ is observed for the BST-MBO varactor over pure BST. However, the interference from the strong acoustics can be observed at these frequencies, which not only degrades the $\eta$, but also shows a distinct resonance effect in tunabilities. At $13.2 \mathrm{MHz}$, the $\eta$ is slightly better for the BST-MBO varactor in comparison, but it is expected to improve for bias voltages above $1.1 \mathrm{kV}$, as shown in Figure $9 \mathrm{~b}$ with the dashed line. These dashed tunabilities, in the extracted $\eta$, are extrapolated by using measured data in the model by Chase et al. [21], as shown in

$$
\varepsilon_{r}(V)=\frac{\varepsilon_{r}(0)}{2 \cosh \left[\frac{2}{3} \cdot \sinh ^{-1}\left(\frac{2 V}{V_{2}}\right)\right]-1},
$$

where $\varepsilon_{r}(V)$ is the calculated permittivity at bias voltage $V, \varepsilon_{r}(0)$ is the unbiased permittivity and $V_{2}$ is the bias voltage at which $50 \%$ tunability is achieved. $V_{2}$ is the fitting parameter from the extracted measured values of relative permittivities between $0 \mathrm{~V}$ and $1.1 \mathrm{kV}$, which is around $1.9 \mathrm{kV}$ and $4.1 \mathrm{kV}$ for the pure BST and BST-MBO varactor, respectively. However, the minimum Q-factors are set as identical to those in the Figure 8a, producing 
the best-case scenario for the pure BST varactor at the bias voltages above $1.1 \mathrm{kV}$. Although $Q_{\varepsilon}$ does not show any noticeable acoustic degradation for the BST-MBO varactor, this may appear when increasing the voltage from $1.1 \mathrm{kV}$ to $4 \mathrm{kV}$, which is also not considered while calculating the extrapolated $\eta$.

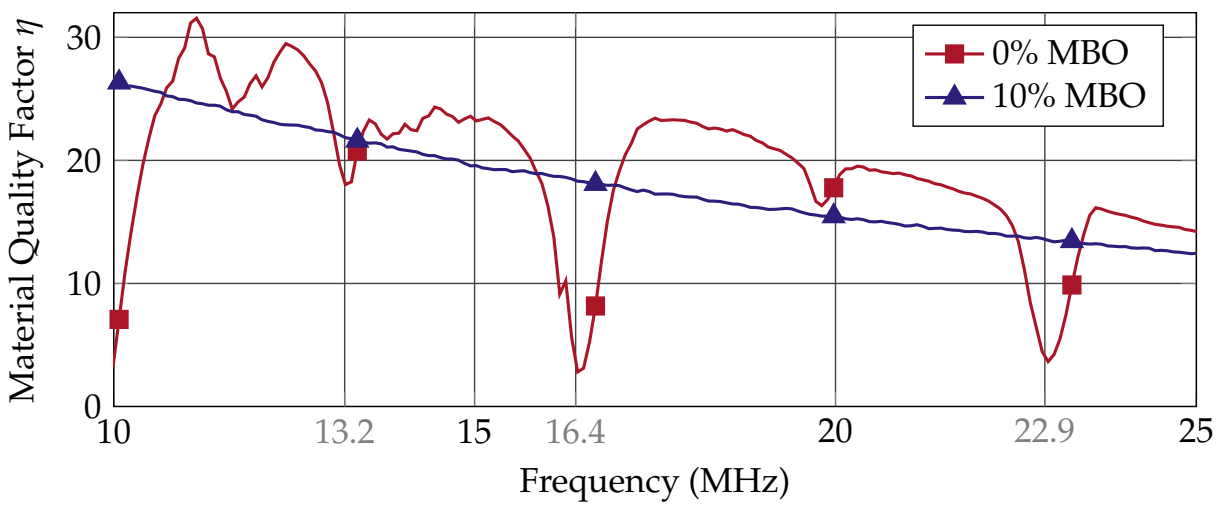

(a)

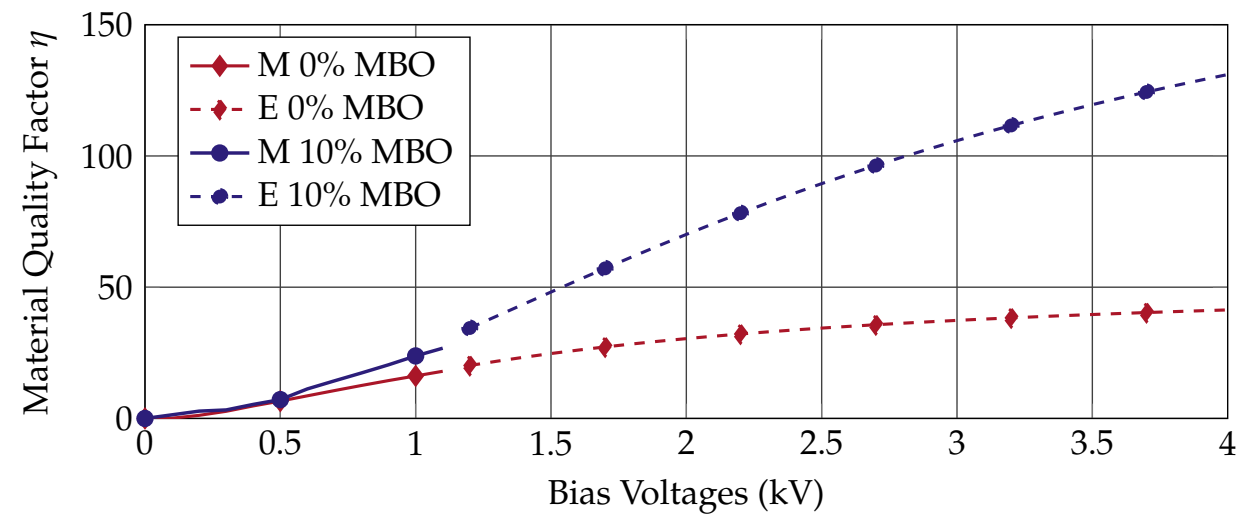

(b)

Figure 9. Material quality factor $(\eta)$ : (a) extracted from measurements at $1.1 \mathrm{kV}(\mathbf{b})$ extracted from measurements $(\mathrm{M})$ and extracted from Chase model (E) at $13.2 \mathrm{MHz}$.

\section{Conclusions}

Quasi-complete suppression of acoustic resonances is achieved in a BST-MBO varactor at the cost of tunability due to decreased amount of tunable material BST. The minimum Q-factor increases from around 60 to 250 at $13.2 \mathrm{MHz}$, but the tunability reduces from $30 \%$ to $10 \%$, with a slightly better material quality factor $(\eta)$ for bias voltages up to $1.1 \mathrm{kV}$. At $16.4 \mathrm{MHz}$ and $22.9 \mathrm{MHz}, \eta$ is notably higher for the BST-MBO varactor. The trade-off between the Q-factor and the capacitance tunability is beneficial for low-loss varactors, where the tunability remains at an acceptable level. In future, a thorough investigation into the incorporation of microstructure changes with the addition of MBO in the BSTbased varactors will be made in the simulations, as well as in experiments, to optimize the performance of the BST-MBO varactors.

Author Contributions: Conceptualization, P.A., D.K., H.M., T.F. and M.A.; methodology, P.A., D.K.; software, P.A., D.K.; validation, J.R.B., H.M., M.A. and T.F.; formal analysis, T.F., K.H. and N.B.; investigation, P.A., N.B. and K.H.; resources, R.J., J.R.B. and W.B.; data curation, P.A.; writingoriginal draft preparation, P.A.; writing-review and editing, H.M., S.M., D.W. and R.J.; visualization, P.A., S.M. and D.W.; supervision, H.M. and R.J.; project administration, H.M., R.J., J.R.B., T.F., W.B. and M.A.; funding acquisition, R.J., T.F. and W.B. All authors have read and agreed to the published version of the manuscript. 
Funding: This work was funded by the Deutsche Forschungsgemeinschaft (DFG) within no. JA921/373 and BI 1636/2-3, COMET AG and the Open Access Publishing Fund of Technical University of Darmstadt.

Institutional Review Board Statement: Not applicable.

Informed Consent Statement: Not applicable.

Data Availability Statement: Contact the authors directly for the data of the presented graph or any other data used in this article.

Conflicts of Interest: The authors declare no conflict of interest.

Sample Availability: Sample varactors were manufactured by the authors from Karlsruhe Institute of Technology.

\section{References}

1. Fang, F. Towards atomic and close-to-atomic scale manufacturing. Int. J. Extrem. Manuf. 2012, 1, 012001. [CrossRef]

2. Kanarik, K.J.; Tan, S.; Gottscho, R.A. Atomic Layer Etching: Rethinking the Art of Etch. Int. J. Phys. Chem. Lett. 2018, 9, 4814-4821. [CrossRef] [PubMed]

3. Chen, Y. Tunable Decoupling and Matching Concepts for Compact Mobile Terminal Antennas. Ph.D. Dissertation, Gottfried Wilhelm Leibniz Universität Hannover, Hannover, Germany, 2017.

4. Andersson, C.M.; Ejebjork, N.; Henry, A.; Andersson, S.; Janzen, E.; Zirath, H.; Rorsman, N. A SiC Varactor With Large Effective Tuning Range for Microwave Power Applications. IEEE Electron Device Lett. 2011, 32, 788-790. [CrossRef]

5. Streeter, R.D.; Hall, C.A.; Wood, R.; Mahadevan, R. VHF High-Power Tunable RF Bandpass Filter Using Microelectromechanical (MEM) Microrelays. Int. J. RF Microw. Comput. Aided Eng. 2001, 11, 261-275. [CrossRef]

6. Preis, S.; Wiens, A.; Kienemund, D.; Kendig, D.; Maune, H.; Jakoby, R.; Heinrich, W.; Bengtsson, O. Discrete RF-power MIM BST thick-film varactors. In Proceedings of the European Microwave Conference (EuMC), Paris, France, 7-10 September 2015; pp. 941-944.

7. Kienemund, D.; Walk, D.; Bohn, N.; Binder, J.R.; Jakoby, R.; Maune, H. Proceedings of the 48th European Microwave Conference Suppression of Acoustic Resonances in Fully-Printed, BST Thick Film Varactors Utilizing Double MIM Structures. In Proceedings of the 48th European Microwave Conference, Madrid, Spain, 23-27 September 2018; pp. 567-570.

8. Jeon, J.H. Effect of $\mathrm{SrTiO}_{3}$ concentration and sintering temperature on microstructure and dielectric constant of $\mathrm{Ba1}-\mathrm{xSrxTiO}$. J. Eur. Ceram. Soc. 2004, 24, 1045-1048. [CrossRef]

9. Nguyen, Q.M.; Anthony, T.K.; Zaghloul, A.I. Free-Space-Impedance-Matched composite dielectric metamaterial with high refractive index. IEEE Antennas Wirel. Propag. Lett. 2019, 18, 2751-2755. [CrossRef]

10. Zhang, Q.; Zhai, J.; Yao, X. Dielectric and Percolative Properties of $\mathrm{Ba}_{0.5} \mathrm{Sr}_{0.5} \mathrm{TiO}_{3}-\mathrm{Mg}_{3} \mathrm{~B}_{2} \mathrm{O}_{6}$ Composite Ceramics. J. Am. Ceram. Soc. 2011, 94, 1138-1142. [CrossRef]

11. Giere, A.; Schafranek, R.; Zheng, Y.; Maune, H.; Sazegar, M.; Jakoby, R.; Klein, A. Characterization of Acoustic Effects in Ferroelectric Thin-Films for Microwave Components. Frequenz 2008, 62, 52-56. [CrossRef]

12. Ghalem, A.; Huitema, L.; Crunteanu, A.; Rammal, M.; Trupina, L.; Nedelcu, L.; Banciu, M.G.; Dutheil, P.; Constantinescu, C.; Marchet, P.; et al. Electrical transport properties and modelling of electrostrictive resonance phenomena in $\mathrm{Ba}_{2 / 3} \mathrm{Sr}_{1 / 3} \mathrm{TiO}_{3}$ thin films. J. Appl. Phys. 2016, 120, 184101. [CrossRef]

13. Newnham, R.E.; Sundar, V.; Yimnirun, R.; Su, J.; Zhang, Q.M. Electrostriction: Nonlinear electromechanical coupling in solid dielectrics. J. Phys. Chem. 1997, 101, 10141. [CrossRef]

14. Li, F.; Jin, L.; Xu, Z.; Zhang, S. Electrostrictive effect in ferroelectrics: An alternative approach to improve piezoelectricity. Appl. Phys. Rev. 2014, 1, 011103. [CrossRef]

15. Gevorgian, S.; Vorobiev, A.; Lewin, T. DC field and temperature dependent acoustic resonances in parallel-plate capacitors based on srtio3 and ba $\mathrm{b}_{0.25} \mathrm{sr}_{0.75} \mathrm{tio}_{3}$ films: Experiment and modeling. J. Appl. Phys. 2006, 99, 124112. [CrossRef]

16. Kienemund, D.; Fink, T.; Abrecht, M.; Bigler, W.; Binder, J.R.; Jakoby, R.; Maune, H. A fully-printed, BST MIM varactor for low ISM-band matching networks up to 1000 W. In Proceedings of the 47th European Microwave Conference, Nuremberg, Germany, 10-12 October 2017; pp. 1001-1004.

17. Abomostafa, H.; Ellamey, M. Studying the mechanical properties of barium strontium titanate ceramics by an ultrasonic pluse echo technique. J. Ovonic Res. 2018, 14, 307-316.

18. Wiens, A.; Kohler, C.; Hansli, M.; Schuessler, M.; Jost, M.; Maune, M.; Binder, J.R.; Jakoby, R. CAD-assisted modeling of high dielectric contrast composite materials. J. Eur. Ceram. Soc. 2017, 37, 1487-1494. [CrossRef]

19. Shen, S.; Xin, L.; Ren, L.; Wang, W.; Jin, J.; Zhai, J.; Zhang, M. Effects of non-stoichiometric defects on the dielectric properties of $\left(\mathrm{Ba}_{0.5} \mathrm{Sr}_{0.5}\right) \times \mathrm{TiO}_{3}-\mathrm{ZnGa}_{2} \mathrm{O}_{4}$ composite ceramics. Ceram. Int. 2020, 46, 22391-22396. [CrossRef] 
20. Giere, A. Material- und Bauteiloptimierung Steuerbarer Mikrowellenkomponenten Mit Nichtlinearen Ferroelektrika. Ph.D. Dissertation, Technical University of Darmstadt, Darmstadt, Germany, 2009.

21. Chase, D.R.; Chen, L.; York, R.A. Modeling the capacitive nonlinearity in thin-film BST varactors. IEEE Trans. Microw. Theory Tech. 2005, 53, 3215-3220. [CrossRef] 